\title{
Neutrino Astrophysics in the cold: Amanda, Baikal and IceCube
}

\author{
Christian Spiering * \\ DESY, Platanenallee 6, D-15738 Zeuthen, Germany
}

\begin{abstract}
This talk reviews status and results from the two presently operating underwater/ice neutrino telescopes, NT-200 in Lake Baikal and AMANDA-II at the South Pole. It also gives a description of the design and the expected performance of IceCube, the next-generation neutrino telescope at South Pole.
\end{abstract}

\section{Introduction}

More than four decades after deep water Cherenkov telescopes for high energy neutrinos have been proposed 1], two detectors of this type are successfully taking data: NT-200 in Lake Baikal, and AMANDA-II at the South Pole. First components of AMANDA's follow-up project of cubic-kilometer size, IceCube, have been deployed in January 2005.

The science topics of these projects include the search for steady and variable sources of high energy neutrinos like Active Galactic Nuclei (AGN), Supernova Remnants (SNR) or microquasars, as well as the search for neutrinos from burst-like sources like Gamma Ray Bursts (GRB) [2]. Underwater/ice telescopes can also be used to tackle a series of questions besides high energy neutrino astronomy. These include the search for neutrinos from the decay of dark matter particles (WIMPs) and the search for magnetic monopoles or other exotic particles - like strange quark matter or Q-balls (see for reviews [3] 4). All these topics are addressed not only by Baikal/NT-200, AMANDA and IceCube, but also by the Mediterranean projects reviewed in the talk of J.J. Aubert at this conference [5].

For deep ice detectors there are two modes of operation which are not - or nearly not - accessible for detectors in natural water. Firstly, due to the low light activity of the surrounding medium, the photomultiplier (PMT) count rate in ice is only about $1 \mathrm{kHz}$. This enables the detection of the feeble increase of individual PMT rates as caused, for instance, by multiple interactions of Supernova burst neutrinos over time intervals of a few seconds. Amanda-II is actually monitoring about $90 \%$ of the Galaxy for MeV neutrinos from Supernova explosions. Secondly, deep ice arrays can be operated in coincidence with surface air shower arrays [6]. This allows one to study questions like the mass composition of cosmic rays up to $10^{18} \mathrm{eV}$, to calibrate the neutrino telescope, and to use the surface detector as a veto against background events.

This paper is organized as follows: In section 2, the design and performance of the two running large neutrino telescopes are sketched, NT-200 in Lake Baikal and AMANDA-II at South Pole. Section 3 is a synopsis of the physics results obtained so far by both detectors (for recent summaries see the talks at the Neutrino 2004 conference [7, 8, 9]. Section 4 desribes design and expected performance of IceCube.

\footnotetext{
${ }^{*}$ Email address: christian.spiering@desy.de
} 


\section{Design of NT-200 and AMANDA}

Underwater/ice neutrino telescopes consist of a lattice of photomultipliers (PMs) housed in transparent glass spheres which are spaced over a large volume in the Ocean, in lakes (like the Baikal telescope) or in ice (as AMANDA and IceCube). The PMs record arrival time and amplitude of the Cherenkov light emitted by muons or particle cascades.

The Baikal Neutrino Telescope NT-200 is operated in Lake Baikal, Siberia, at a depth of $1.1 \mathrm{~km}$. An umbrella-like frame carries 8 strings, each with 24 optical modules (OMs) arranged in pairs. The pressure glass spheres of the OMs contain 37-cm diameter PMs. The instrumented volume forms a cylinder of $70 \mathrm{~m}$ height and $42 \mathrm{~m}$ diameter. The angular resolution for through-going muons is about $4^{\circ}$.

The present, final configuration of the AMANDA neutrino telescope is named AMANDA-II. It consists of 677 OMs arranged along 19 vertical strings buried in the glacial ice at the South Pole, with most of the OMs at depths between 1500 and $2000 \mathrm{~m}$. The geometric shape of the array is a cylinder $\sim 500 \mathrm{~m}$ high and $\sim 200 \mathrm{~m}$ in diameter. Each AMANDA OM houses an 8-inch Hamamatsu photomultiplier. The present angular resolution for muons tracks is about $2.5^{\circ}$. Figure 1 sketches the configuration of both detectors.

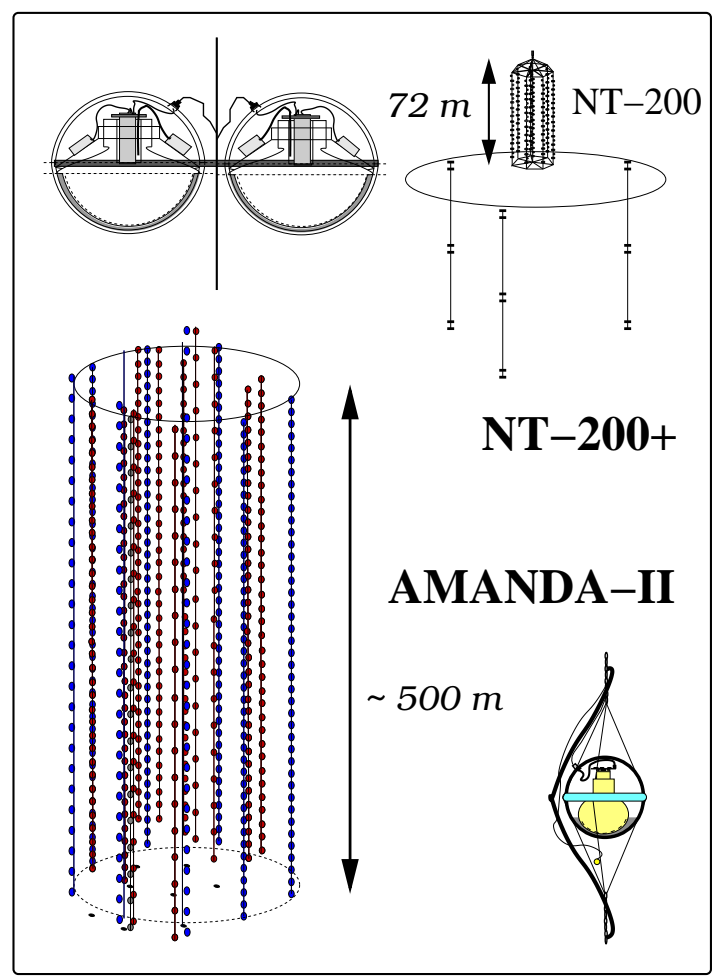

Figure 1: AMANDA-II and NT-200 (NT200+). For AMANDA-II, only the central part between 1500 and 2000 meters is shown, covering about $90 \%$ of the optical modules. Detectors are shown to scale. Also sketched are the optical modules. AMANDA PMs have $20 \mathrm{~cm}$, Baikal PMs $37 \mathrm{~cm}$ diameter.

NT-200 takes data since April 1998. It has been deployed in subsequent stages, starting in 1993 with NT-36, the pioneering first stationary underwater array [10]. Components are deployed from an natural fixed platform: the thick ice layer which covers Lake Baikal in February and March, when uutside temperatures 
reach down to $-25^{\circ}$ (see for more technical details [12]). Figure 2(left) shows a textbook neutrino event (an upward moving muon track) recorded with the early 4-string configuration of 1996 [1].

The absorption length of deep Baikal water varies between 20 and 24 meters. Since light scattering is small, NT-200 can monitor a volume exceeding its own geometric volume by an order of magnitude. Actually, this is the reason that the sensitivity of NT-200 with respect to high energy, diffuse-flux phenomena is not dwarfed by that of the much larger AMANDA-II (AMANDA is embedded in ice where light scattering is strong and diffuses light from distant sources). In 2005, NT-200 is going to be upgraded with three sparsely instrumented outer strings (NT-200+, see Fig.1). The three strings will allow a dramatically improved vertex reconstruction of high energy cascades within this volume and will increase the sensitivity to diffuse fluxes by a factor of four (see section 3 ).

Rather than water, AMANDA uses ice as detection medium. The glacial ice is extremely transparent for Cherenkov light with wavelengths near the peak sensitivity of the OMs: At $400 \mathrm{~nm}$, the average absorption length is $110 \mathrm{~m}$. Scattering, however, is much stronger than in water - the average effective scattering length is only $20 \mathrm{~m}$. Below a depth of $1500 \mathrm{~m}$, both scattering and absorption are dominated by dust, and the optical properties vary with dust concentration.

To deploy AMANDA, holes were melted with hot water, and strings with OMs frozen into the ice. Similar to the Baikal telescope, AMANDA was also deployed step by step. An earlier 10-string stage, called AMANDA-B10, was installed between 1997 and 1999 [13. First neutrinos have been recorded with the inner four strings deployed in 1996 (see Fig. 2, right). AMANDA-II has been taking data since 2000.
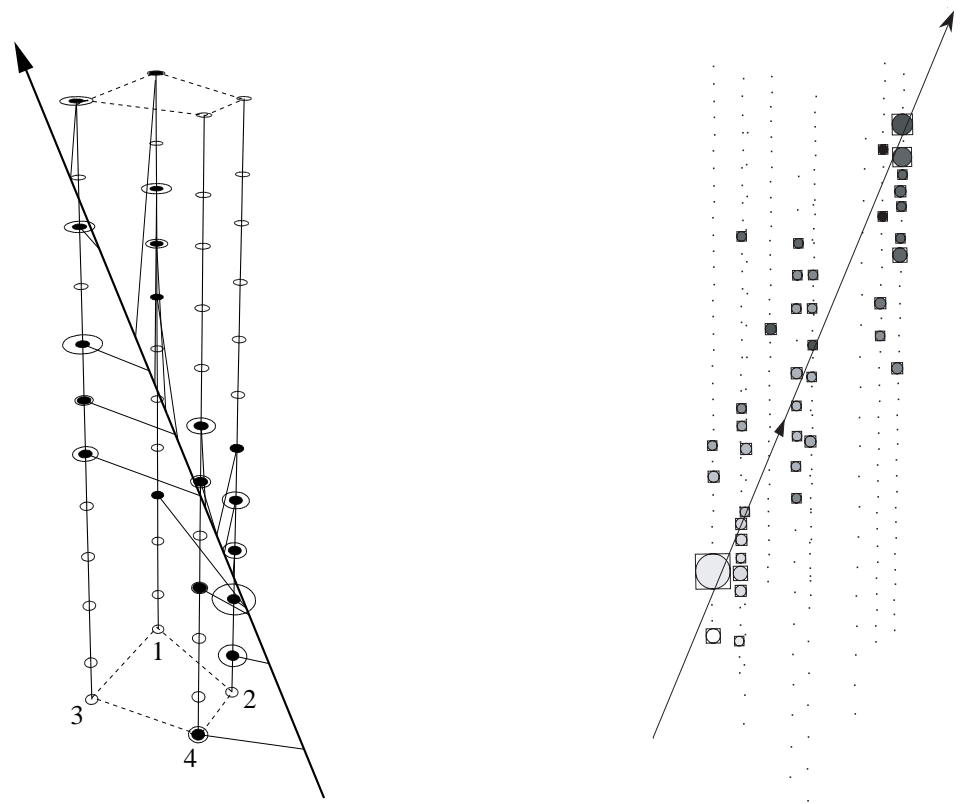

Figure 2: Left: one of the first clearly upward moving muons recorded with the 1996 four-string-stage of the Baikal detector. Black dots denote PMs. Hit PMs are encircled, with the size of the disc proportional to the recorded amplitude. The arrow line represents the reconstructed muon track, the thin lines the photon paths. Right: Upward muon recorded by the 1997 stage of AMANDA. Dots denote the PMs arranged at ten strings. Hit PMs are highlighted, with the degree of shadowing indicating the arrival time (dark being late), and the size of the symbols the measured amplitude. Note the different vertical scales for these events: $70 \mathrm{~m}$ for NT-200, $500 \mathrm{~m}$ for AMANDA. 


\section{Physics results from NT-200 and AMANDA}

\subsection{Proof of principle: atmospheric neutrinos}

For most analysis channels, both AMANDA and NT-200 use the Earth as a filter and separate up-going muons steming from interactions of neutrinos having crossed the Earth. The main class of background are down-going atmospheric muons that are misreconstructed as up-going. After their rejection, basically upgoing muons from interactions of neutrinos generated in the atmosphere remain. Atmospheric neutrinos not only constitute the main background when searching for extraterrestrial neutrinos, but are also a natural calibration source. Figure 3 shows the energy spectrum for up-going neutrinos based on AMANDA-II data taken in 2000. It has been obtained by a neural net energy reconstruction, followed by regularized unfolding. This is the first atmospheric neutrino spectrum above a few $\mathrm{TeV}$, and it extends up to $300 \mathrm{TeV}$.

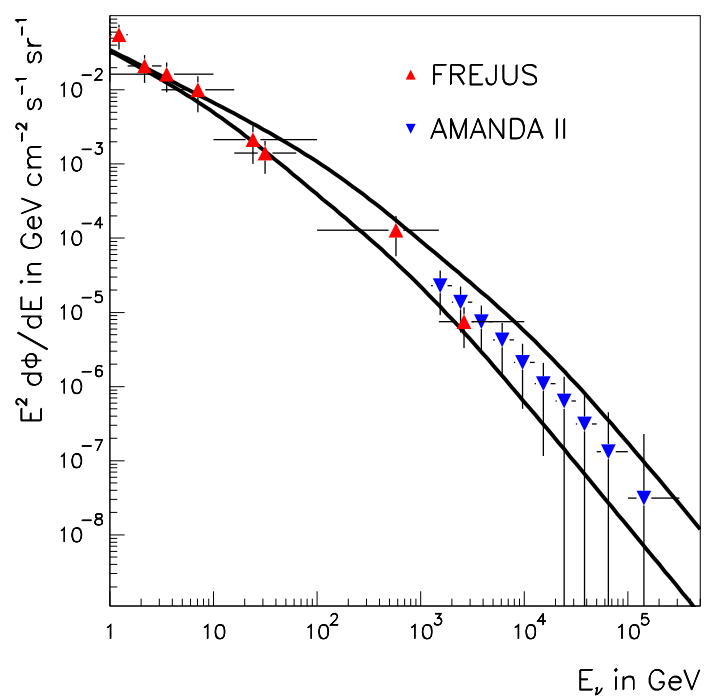

Figure 3: Atmospheric neutrino energy spectrum (preliminary) from regularized unfolding of AMANDA data, compared to the Frejus spectrum [14] at lower energies. The two solid curves indicate model predictions [15] for the horizontal (upper) and vertical (lower) flux.

AMANDA, with its $60 \mathrm{GeV}$ threshold for the standard event selection, is not sensitive to neutrino oscillations. The threshold of the Baikal telescope NT-200 is much lower and, for tight selection procedures, can be reduced down to $10 \mathrm{GeV}$. Figure 4 shows the angular spectrum for upward tracks close to the opposite zenit. The $20 \%$ deficit close to the vertical is well compatible with the the oscillation parameters $\delta m^{2} \approx 2.5 \cdot 10^{-3}$ and $\sin ^{2} \theta \approx 1$. This effect has to be taken into account when using these data as standard signal for detector calibration.

\subsection{Search for a diffuse flux of cosmic neutrinos}

The primary destination of neutrino telescopes is the identification of individual, point-like sources of highenergy neutrinos. Should individual sources be too weak to produce an unambiguous directional signal in the array, the integrated neutrino flux from all sources could still produce a detectable diffuse signal. This flux 


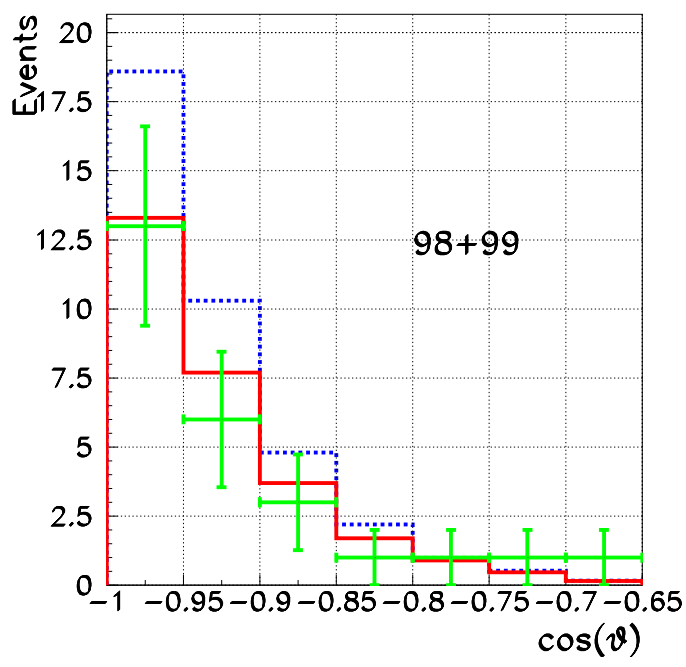

Figure 4: Angular spectrum of nearly vertical atmospheric neutrinos recorded with NT-200 in 1998/99. $\cos \theta=-1$ corresponds to vertically upward moving tracks. Data are compared to simulated distributions including (full line) and excluding (dotted line) the effect of oscillations.

could be revealed by an high energy excess on top of the omni-present background of atmospheric neutrinos. The data of both experiments have been searched for such a diffuse signal using complementary techniques in different energy regimes. Event selection is typically optimized to maximize the sensitivity to an $E^{-2}$ signal spectrum. Experimental limits given below include statistical as well as systematic errors (the latter being typically between 20 and 40 percent).

Upward moving muons: The atmospheric neutrino spectrum recorded with AMANDA-II (Fig. 3) was used to set an upper limit on a diffuse $E^{-2}$ flux of extraterrestrial muon neutrinos for the energy range covered by the highest bin, $100-300 \mathrm{TeV}$, by calculating the maximal non-atmospheric contribution to the flux in that bin given its statistical uncertainty. However, the bins in the unfolded spectrum are correlated and the uncertainty in the last bin can not a priori be assumed to be Poissonian. The statistics in the bin was therefore determined with many Monte Carlo samples used to construct confidence belts. Given the (fractional) unfolded number of experimental events in the bin, a preliminary 90\% C.L. upper limit of $E^{2} \Phi_{\nu_{\mu}}(E)<2.6 \times 10^{-7} \mathrm{~cm}^{-2} \mathrm{~s}^{-1} \mathrm{sr}^{-1} \mathrm{GeV}$ is derived for $100 \mathrm{TeV}<E_{\nu}<300 \mathrm{TeV}$.

Cascades: Apart from muon tracks, cascades can be detected. With a typical length of 5-10 m and a diameter of $10 \mathrm{~cm}$, cascades can be considered as quasi point-like compared to the spacing of OMs. All three neutrino flavors contribute to this signature - cascades stem from the leptonic vertex of electron and tau neutrino charged current interactions, and hadronic vertex cascades from all-flavor neutral current interactions.

The AMANDA analysis focuses on contained events (allowing good energy reconstruction), the Baikal/NT200 survey to bright cascades produced at the neutrino interaction vertex in a large volume around the neutrino telescope. (As pointed out in the introduction, lack of significant light scattering allows to monitor a volume exceeding the geometrical volume of NT-200 by an order of magnitude.) 
Both analyses did not yield any excess of candidate events over background: 1 observed event (vs. 0.9 background events) in the case of the year-2000 AMANDA data, no event (vs. 0.4 background events) in the case of the 1998-2003 NT-200 data. The corresponding upper limits with respect to the flux of all three flavors are $\Phi_{\nu_{e}+\nu_{\mu}+\nu_{\tau}} E^{2}=8.6 \cdot 10^{-7} \mathrm{~cm}^{-2} \mathrm{~s}^{-1} \mathrm{sr}^{-1} \mathrm{GeV}$ (AMANDA, $50 \mathrm{TeV}<E_{\nu}<5 \mathrm{PeV}$ [18]) and $\Phi_{\nu_{e}+\nu_{\mu}+\nu_{\tau}} E^{2}=8.1 \cdot 10^{-7} \mathrm{~cm}^{-2} \mathrm{~s}^{-1} \mathrm{sr}^{-1} \mathrm{GeV}$ (NT-200, $22 \mathrm{TeV}<E_{\nu}<50 \mathrm{PeV}$ [19]), calculated under the assumption that neutrinos arrive with a ratio $\nu_{e}: \nu_{\mu}: \nu_{\tau}=1: 1: 1$.

Ultra High Energy neutrinos: At ultra-high energies (UHE), above $1 \mathrm{PeV}$, the Earth is opaque to electron- and muon-neutrinos. The AMANDA search for extraterrestrial UHE neutrinos is therefore concentrated on events close to the horizon and even from above. The latter is possible since the atmospheric muon background is low at these high energies due to the steeply falling energy spectrum. The search for UHE events in 1997 AMANDA-B10 data relies on parameters that are sensitive to the expected characteristics of an UHE signal: bright events, long tracks (for muons), low fraction of single photoelectron hits. No excess above background is observed and a $90 \%$ C.L. limit on an $E^{-2}$ flux of neutrinos of all flavors is derived, assuming a 1:1:1 flavor ratio at Earth [20]: $\Phi_{\nu_{e}+\nu_{\mu}+\nu_{\tau}} E^{2}<9.9 \times 10^{-7} \mathrm{~cm}^{-2} \mathrm{~s}^{-1} \mathrm{sr}^{-1} \mathrm{GeV}\left(1 \mathrm{PeV}<E_{\nu}<3 \mathrm{EeV}\right)$.

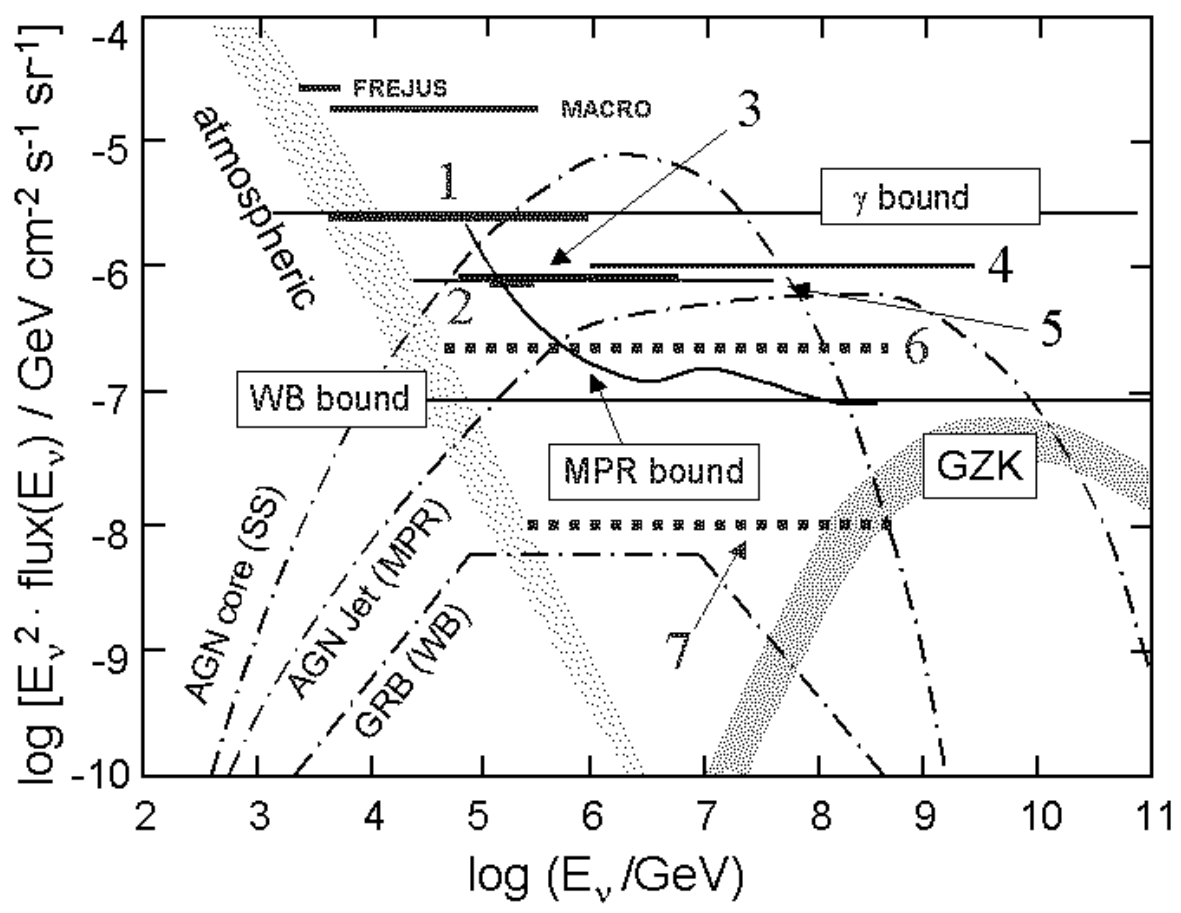

Figure 5: Theoretical and experimental limits and model predictions to the diffuse flux of extraterrestrial neutrinos, compared to the flux of atmospheric neutrinos. 1) AMANDA-B10, upward muons [16], 2) AMANDA-II, upward muons (prelim.), 3) AMANDA-II, cascades [18, 4) AMANDA-B10, UHE events [20], 5) NT-200, cascades (prelim. [19]) 6) AMANDA-II and NT200+: expectation for 4 years, 7) IceCube expectation for 3 years. Shown are the limits to the flux of all flavors, with the assumption that $\nu_{e}: \nu_{\mu}: \nu_{\tau}=1: 1: 1$ at Earth. Experimental limits obtained for muons alone have been multiplied by a factor of 3 . Theoretical bounds and predictions which have been originally given for muons and assuming $\nu_{e}: \nu_{\mu}: \nu_{\tau}=1: 2: 0$ (i.e.without consideration of oscillations) have been multiplied by a factor 1.5. For the MPR and WB bound see [22, 23]. 
Summary of diffuse searches: Using different analysis techniques, AMANDA and NT-200 yield limits on the diffuse flux of neutrinos with extraterrestrial origin for neutrino energies from $6 \mathrm{TeV}$ up to a few $\mathrm{EeV}$. Figure 5 5ummarizes the flux predictions, upper bounds derived from observed fluxes of charged cosmic rays and gamma rays, and existing best limits of AMANDA and NT-200 as well as extrapolations to several years of AMANDA data and to IceCube. With the exception of the limit from the unfolded atmospheric spectrum, which can be seen as a quasi-differential limit, the limits are on the integrated flux over the energy range which contains $90 \%$ of the signal. These limits exclude some models like 21. Note that both experiments enter new territory, being below the gamma bound [17] where sensitivities are not a priori too weak to hope for a discovery (see also section 4.2).

\subsection{Search for point sources}

Searches for neutrino point sources require good pointing resolution and are thus restricted to the $\nu_{\mu}$ channel. Both experiments have produced sky-plots based on data taken over several years. Figure 6 shows 3329 upward moving muons recorded by AMANDA during four years (2000-2003), and 372 Baikal events recorded during 5 years (1998-2003). Since good pointing resolution is mandatory, and since this can be achieved only for tracks crossing the array, the smaller NT-200 cannot compete with AMANDA. We note, however, that for hard neutrino spectra (e.g. $E^{-2}$ ) NT-200 is yet the largest telescope at the Northern hemisphere and nicely complements AMANDA in the South. In the following, only AMANDA data will be discussed in more detail.

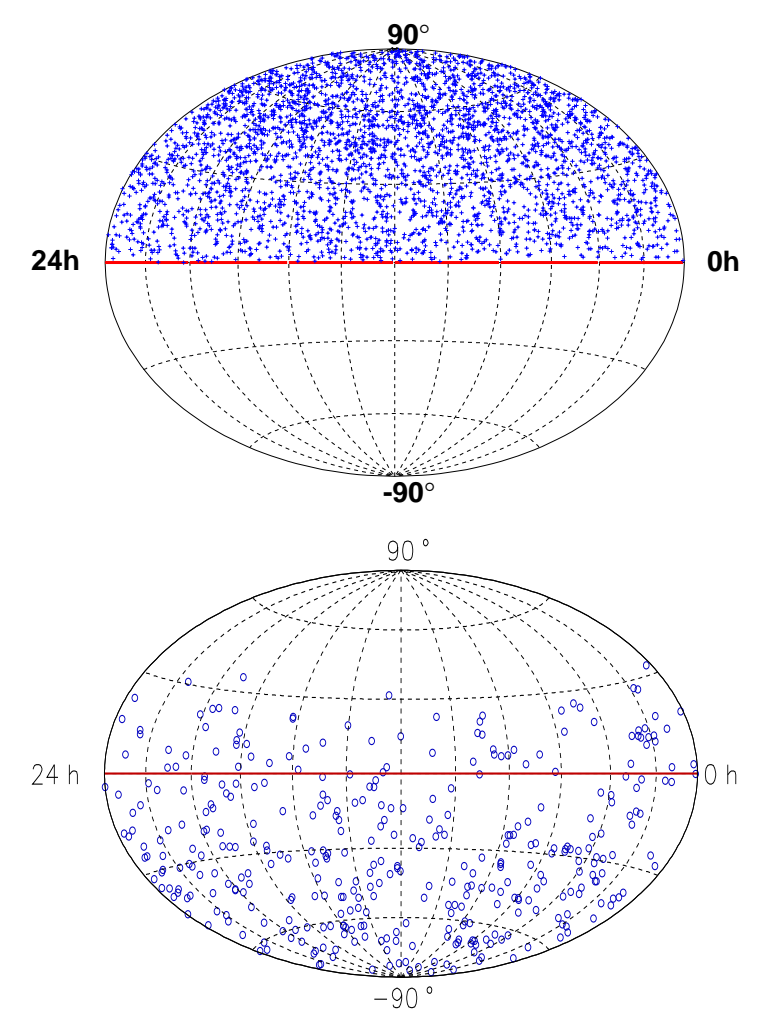

Figure 6: Sky map in equatorial coordinates. Top: 3329 neutrino candidates recorded with AMANDA-II in 2000-2003. Bottom: 372 neutrino candidates recorded with NT-200 in 1998-2003 (preliminary). 
AMANDA events were selected to maximize the model rejection potential for an $E^{-2}$ neutrino spectrum convoluted with the background spectra due to atmospheric neutrinos and misreconstructed atmospheric muons. The sensitivity of the analysis, defined as the average upper limit one would expect to set on a nonatmospheric neutrino flux if no signal is detected, is about $E^{2} \Phi_{\nu}(E)<0.6 \times 10^{-7} \mathrm{~cm}^{-2} \mathrm{~s}^{-1} \mathrm{GeV}_{\text {, averaged }}$ over the lower hemisphere and for a hypothetical $E^{-2}$ signal spectrum. This is three times lower than the one-year limit for the year 2000 [24]. It corresponds to a neutrino flux above $1 \mathrm{TeV}$ of $F_{\nu}(>1 \mathrm{TeV})<6 \cdot 10^{-10}$ $\mathrm{cm}^{-2} \mathrm{~s}^{-1}$ - about a factor five above the Crab gamma flux and close to the gamma flux from Markarian-501 in its flaring phase [25].

The final sample of 3369 neutrino candidates (3329 from below, with 3438 expected atmospheric neutrinos) was searched for point sources with two methods. In the first, the sky is divided into a fine-meshed grid of overlapping bins which are tested for a statistically significant excess over the background expectation (estimated from all other bins in the same declination band). This search yielded no evidence for extraterrestrial point sources. The second method is an unbinned search, in which the sky locations of the events and their uncertainties from reconstruction are used to construct a sky map of significance in terms of fluctuation over background. The hot spots on this map are well within the expectation from a random event distribution. In a search for 33 preselected sources, the strongest excess was observed from the direction of the Crab nebula, with 10 events where 5 are expected - again no significant effect given the number of trials. One thus sees no evidence for point sources with an $E^{-2}$ energy spectrum based on the first four years of AMANDA-II data, and it seems unlikely that another few years of data will change this result. Obviously, a much larger array like IceCube is neccessary to detect steady sources. However, the picture may change principally for transient sources, where, for searches during known gamma flares (like e.g. the blazars Mkr-421, Mkr-501, ES1959+650), the signal-to-noise ratio may improve dramatically.

\subsection{Search for neutrinos from GRBs}

A special case of point source analysis is the search for neutrinos coincident with gamma ray bursts (GRBs) detected by satellite-borne detectors. Here, the timing of the neutrino event serves as an additional selection handle which significantly reduces background.

Both collaborations have used the GRB sample collected by the BATSE satellite detector which was decomissioned in 2000. The AMANDA (Baikal) and BATSE data taking periods were overlapping in 19972000 (1998-2000). Samples containing 312 (368) bursts triggered by BATSE from this period have been analyzed by AMANDA (Baikal). Data were searched for an excess of events in a 10 min (100 second) window around the GRB time. No coincident neutrino event was observed in the case of AMANDA, and one (over a background of 0.46) for Baikal. Assuming a broken power-law energy spectrum as proposed by Waxmann and Bahcall 23, the 90\% C.L. upper limit on the expected neutrino flux at the Earth derived by AMANDA is $E^{2} \Phi_{\nu}(E)<4 \times 10^{-8} \mathrm{~cm}^{-2} \mathrm{~s}^{-1} \mathrm{sr}^{-1} \mathrm{GeV}$. This is approximately a factor 15 above the WaxmannBahcall flux prediction.

Other classes of bursts are being included in the analysis, like the so-called non-triggered BATSE bursts and triggers from the Third Interplanetary Network (IPN3), since 2000 the major source of GRB detection. 


\subsection{Dark matter search}

The Minimal Supersymmetric extension of the Standard Model (MSSM) provides a promising dark matter candidate in the neutralino, which could be the lightest supersymmetric particle. Neutralinos can be gravitationally trapped in massive bodies, and can then via annihilations and the decay of the resulting particles produce neutrinos. Dark matter can therefore indirectly searched for by looking for fluxes of neutrinos from the center of the Earth or the Sun.

Both collaborations have searched for vertically up-going tracks from the center of the Earth, for AMANDA-B10 using data from 1997-1999, for NT-200 using data from 1998/1999. No indication of an excess over atmospheric neutrinos was found. The 90\% C.L. upper limit on the muon flux from the center of the Earth are compared in Fig. $\mathbf{7}$ (left) to limits obtained from underground experiments and to MSSM model predictions excluded by direct search.

With its larger mass and a higher capture rate due to additional spin-dependent processes, the Sun is more effective than the Earth in catching WIMPs. AMANDA-II data from 2001 data yield no indication of a WIMP signature. The preliminary upper limit on the muon flux from the Sun is compared to MSSM predictions in Fig. 7 (right). For heavier neutralino masses, the limit obtained with less than one year of AMANDA-II data is already competitive with limits from indirect searches with detectors that have several years of integrated livetime. It should be noted that the two methods are complementary since they (a) probe the WIMP distribution in the solar system at different epochs and (b) are sensitive to different parts of the velocity distribution. Direct searches are sensitive to high-energy recoils and therefore to the high-velocity tail of the WIMP flux, indirect searches are more sensitive to low-velocity WIMPs since those are easier captured by celestial bodies.
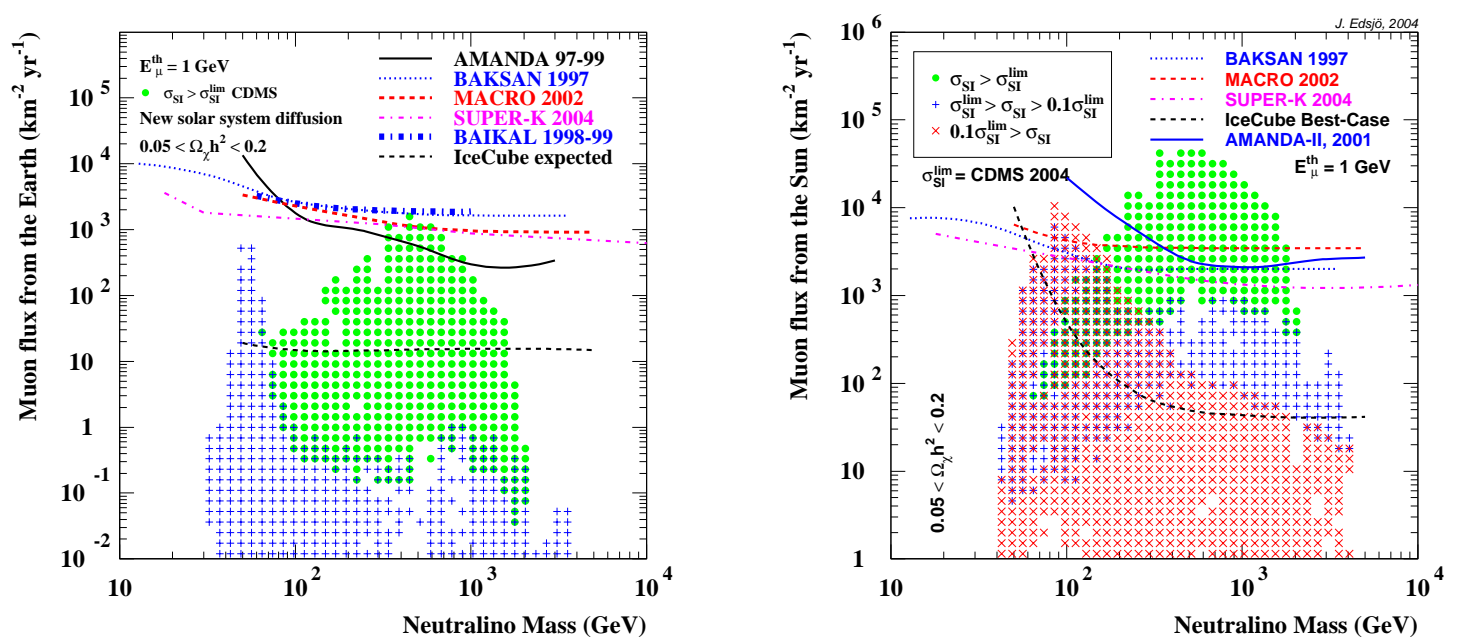

Figure 7: Limits on the muon flux due to neutrinos from neutralino annihilations in the center of the Earth (left) and the Sun (right). The symbols correspond to model predictions [3] within the allowed parameter space of the MSSM. The dots represent models disfavored by direct searches with CDMS II [27]. Crosses $(+)$ cover models which could be tested by the direct searches ten times more sensitive than the present ones. Amanda and Baikal limits are preliminary. 


\subsection{Magnetic monopoles}

A magnetic monopole with unit magnetic Dirac charge $g=137 / 2 \cdot e$ and velocities above the Cherenkov threshold in water $(\beta>0.75)$ would emit Cherenkov radiation smoothly along its path, exceeding that of a bare relativistic muon by a factor of 8300 . This is a rather unique signature. Figure 8 summarizes the limits obtained until now. A cube kilometer detector could improve the sensitivity of this search by nearly two orders of magnitude. The search could be extended to even lower velocities by detection of the $\delta$ electrons generated along the monopole path.

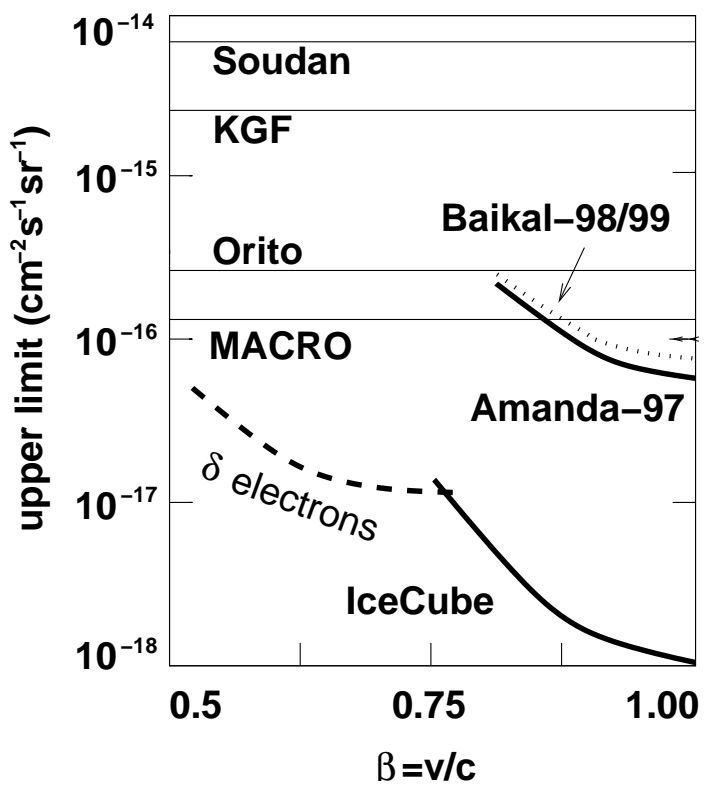

Figure 8: Upper limits on the flux of relativistic monopoles obtained in different experiments.

Limits on the flux of particles moving with with less than $10^{-3} c$, like GUT magnetic monopoles catalyzing baryon decay, Q-balls or nuclearites [4] have been obtained with early stages of the Baikal detector [10, 28. The trigger system of the future Icecube is flexible enough to search effectively for such particles and to lead to much stronger limits than those of [28].

\subsection{Detection of Supernova bursts}

Due to the low external noise rate, AMANDA is sensitive to the increase of individual counting rates of all PMs resulting from a Supernova burst 29. AMANDA-II can detect $90 \%$ of supernovae within $9.4 \mathrm{kpc}$ with less than 15 fakes per year. This is sufficiently robust for AMANDA to contribute to the SuperNova Early Warning System (SNEWS) with neutrino detectors in the Northern hemisphere. IceCube will monitor the full Galaxy. An alarm would confirm other records within SNEWS but also provide directional information: If several detectors spread around the world would measure the signal front with an accuracy of a few ms, one might determine the supernova direction by triangulation. The high statistics of hits recorded by IceCube would allow a precise measurement of the set-in of the burst [30. 


\section{IceCube}

\subsection{The detector}

With 4800 optical modules on 80 strings, horizontally spaced by $125 \mathrm{~m}$, IceCube [9] covers an area of approximately $1 \mathrm{~km}^{2}$, with the OMs at depths of 1.4 to $2.4 \mathrm{~km}$ below surface. Each string carries $60 \mathrm{OMs}$, vertically spaced by $17 \mathrm{~m}$. The strings are arranged in a triangular pattern. The configuration of IceCube is shown in Fig 9] At each hole, one station of the IceTop air shower array [32] will be positioned. An IceTop station consists of two ice tanks of total area $7 \mathrm{~m}^{2}$.

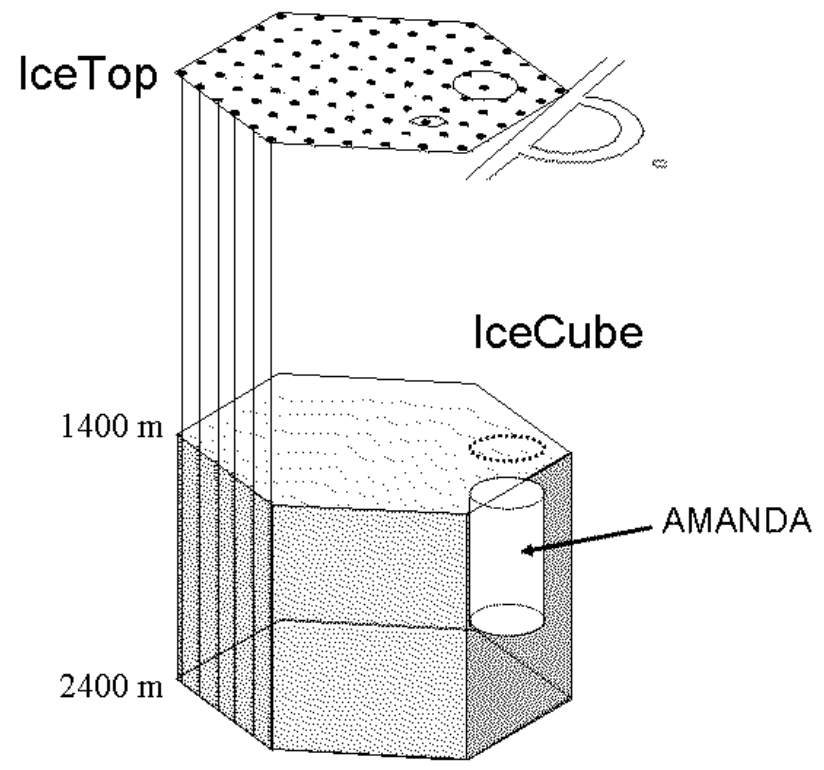

Figure 9: Schematical View of IceCube, IceTop and AMANDA.

A first IceCube string has been successfully deployed on January 27, 2005. For IceCube, a new drilling system has been constructed. The power for heaters and pumps of the EHWD is now $\sim 5 \mathrm{MW}$, compared to $2 \mathrm{MW}$ for AMANDA. This, and the larger diameter and length of the water transporting hoses, results in only 40 hours needed to drill a $2400 \mathrm{~m}$ deep hole (three times faster than with the old AMANDA drill). Mounting, testing and drop of a string with 60 DOMs takes about 20 hours, and deployment of ultimately 18 strings per season seems feasible.

The present AMANDA-II detector will be integrated into IceCube. IceCube will deliver efficient veto information for low energy cascade-like events or short horizontal tracks recorded in AMANDA. Horizontal tracks could be related to neutrinos steming from WIMP annihilations in the Sun.

The IceCube OM contains a 10-inch diameter PM HAMAMATSU R-7081. Different to AMANDA, the PM anode signal is digitized within the $\mathrm{OM}$ and sent to the surface via electrical twisted-pair cables. Waveforms of the signals are recorded with $250 \mathrm{MHz}$ over the first $0.5 \mu \mathrm{s}$ and $40 \mathrm{MHz}$ over $5 \mu \mathrm{s}$. The fine sampling is done with the Analog Transient Waveform Recorder (ATWR), an ASIC with four channels, each capable to capture 128 samples with $200-800 \mathrm{~Hz}$. The $40 \mathrm{MHz}$ sampling is performed by a commercial FADC. Each pulse time stamped with 7 ns r.m.s.. See 31] for more details. 


\subsection{Physics Performance}

Figure 10 shows the IceCube effective area for muons after $\sim 10^{-6}$ reduction of events from downward muons, as a function of the muon zenith angle [33. Whereas at $\mathrm{TeV}$ energies IceCube is blind towards the upper hemisphere, at $\mathrm{PeV}$ and beyond the aperture extends above the horizon and allows observation of the Southern sky.

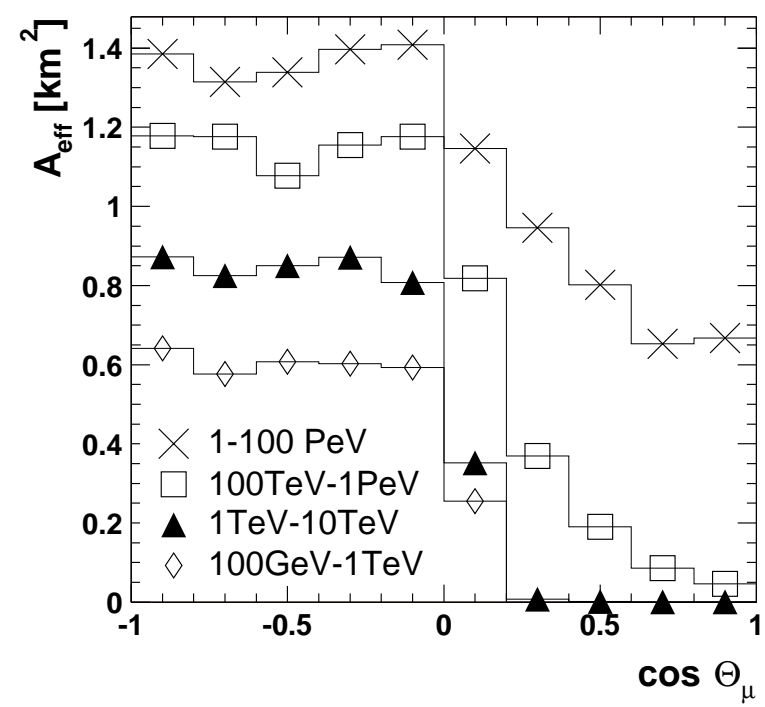

Figure 10: IceCube effective area for muon as a function of zenith angle. $\cos \theta=-1$ denotes vertically upward moving muons, $\cos \theta=0$ marks the horizontal direction.

The IceCube sensitivity to diffuse fluxes after three years of data taking is shown in Fig. 5 (section 3.2). The dashed-dotted lines indicate the Stecker and Salamon model for photo-hadronic interactions in AGN cores [21] and of the model of Mannheim, Protheroe and Rachen on neutrino emission from photo-hadronic interactions in AGN jets 22]. In case of no signal observed, these models could be rejected with model rejection factor of $10^{-3}$ and $10^{-2}$ respectively. Also shown is the GRB estimate by Waxman and Bahcall 23] which would yield of the order of ten events coinciding with a GRB, for 1000 monitored GRBs.

For not too steep angles the IceCube pointing resolution is 0.6 to 0.8 degrees, improving with energy. We expect that evaluation of waveform information will improve these numbers significantly, at least at high energies, and increase the potential for point source identification.

Figure 11 sketches a possible scenario for the point source search over the next decade. Best present limits are from MACRO, Super-Kamiokande (Southern sky) and AMANDA (Northern sky). Baikal limits for the Southern sky will appear soon. This picture will not change until the medium stage Mediterranean detectors come into operation. The ultimate sensitivity for the $\mathrm{TeV}-\mathrm{PeV}$ range is likely reached by the cubic kilometer arrays. This scale is set by many model predictions for neutrinos from cosmic accelerators or from dark matter decay. A discovery with AMANDA is not yet excluded - be it a flaring blazar or a supernova; for IceCube, hundred times larger than AMANDA and thousand times larger than underground detectors, it seems extremely likely. However, irrespective of any specific model prediction, IceCube will hopefully also keep the promise for any detector opening a new window to the Universe: to detect unexpected phenomena. 


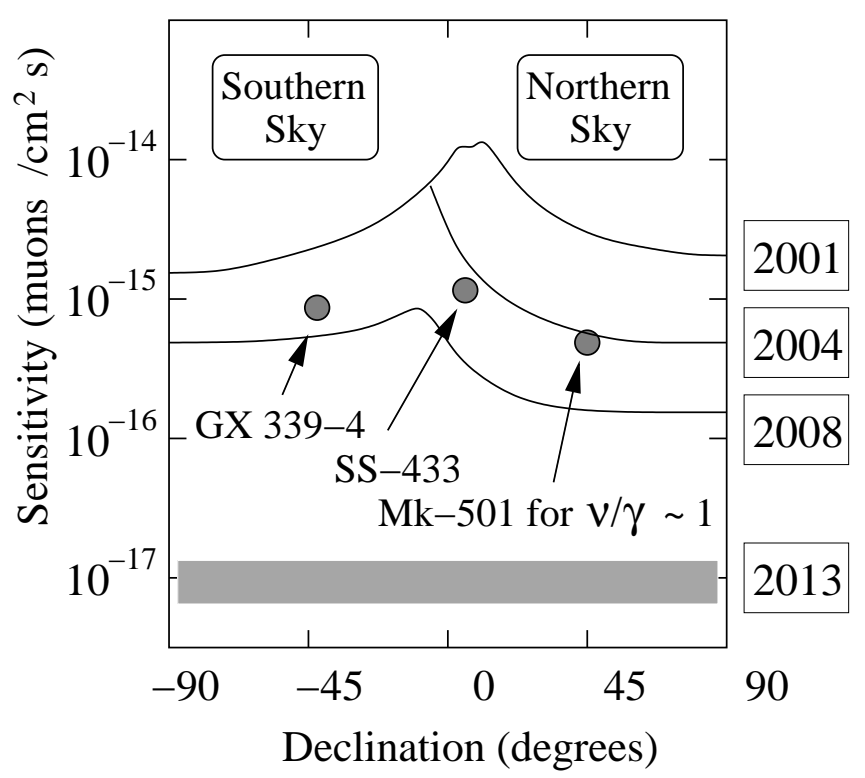

Figure 11: Scenario for the improvement of experimental sensitivities to $\mathrm{TeV}$ point sources. Expected steps for the Northern sky are obtained from Amanda alone and Amanda together with the first 25-30 strings of IceCube, on the Southern sky from the Mediterranean detectors Antares and Nestor (2008). In 2013, both hemispheres will be instrumented with cubic kilometer arrays indicated by the grey band. Shown are also predicted fluxes for two microquasars [35] - one at northern and one on the southern hemisphere - which are just in reach for Amanda and the Mediterranean arrays. As a benchmark, I also show the flux which would be expected if Mk-501, a source spectacular in $\mathrm{TeV}$ gamma rays, would produce a flux of $\mathrm{TeV}$ muon neutrinos similar to that of gammas emitted in its flaring phase.

\section{Acknowledments}

I thank my colleagues in the collaborations Baikal, AMANDA and IceCube for the long, fruitful cooperation and for helpful discussions.

\section{References}

[1] M. Markov, Proc. 1960 Int. Conf. on High Energy Physics, Rochester 1960, 578.

[2] F. Halzen, these proceedings, and F. Halzen and D. Hooper, Rept. Prog. Phys. 65 (2002) 1065.

[3] J. Edsjö, Proc. Int. Conf. Neutrino-2004, Paris, and references therein.

[4] D.Bakari et al., hep-ex/0004019, S. Wick et al., Astropart. Phys. 18 (2003) 663.

[5] J.J. Aubert, these proceedings.

[6] J. Ahrens et al., Astropart. Phys. 21 (2004) 565.

[7] Zh.-A. Dzilkibaev for the Baikal Collaboration, Nucl Phys B(Proc. Suppl.) 143 (2005) 335.

[8] K. Woschnagg for the Amanda Collaboration, ibid. page 343.

[9] O. Botner for the IceCube Collaboration, ibid. page 367. 
[10] I. Belolaptikov et al., Astropart. Phys. 7 (1997) 263.

[11] I. Belolaptikov et al., Astropart. Phys.12 (1999) 75.

[12] C. Spiering for the Baikal Coll., Proc. VLVNT Workshop, Amsterdam 2003, 26, and astro-ph/0404096

[13] E. Andrés et al., Nature 410 (2001) 441.

[14] K. Daum et al., Z. Phys. C 66 (1995) 417.

[15] L. V. Volkova, Sov. J. Nucl. Phys. 31 (1980) 784.

[16] J. Ahrens et al., Phys. Rev. Lett. 90 (2003) 251101.

[17] V.S.Berezinsky and A.Yu. Smirnov, Astrop. Sp. Sci. 32 (1975) 461.

[18] M. Ackermann et al., Astropart. Phys. 22 (2004) 127, astro-ph/0405218

[19] V. Aynutdinov et al., to be published in Proc. 19th ECRS, Florence (2004).

[20] M. Ackermann et al., Astropart. Phys. 22 (2005) 339.

[21] F.W. Stecker and M.H. Salamon, Space Sci. Rev. 75 (1996) 341.

[22] K. Mannheim, R. Protheroe and J. Rachen, Phys. Rev. D63 (2001) 023003.

[23] E. Waxmann and J. Bahcall, Phys. Rev. D59 (1999) 023002.

[24] J. Ahrens et al., Phys. Rev. Lett. 92 (2004) 071102.

[25] see e.g. D. Horan, D.C. Weekes, New Astron. Rev. 48 (2004) 527, and astro-ph/0310391.

[26] J. Ahrens et al., Phys. Rev. D66 (2002) 032006.

[27] D.S. Akerib et al., astro-ph/0405033

[28] L.B. Bezrukov et al., Sov. J. Nucl. Phys. 52 (1990) 54.

[29] J. Ahrens et al., Astropart. Phys. 16 (2002) 345.

[30] T. Neunhöffer for the Amanda Collaboration, Proc. 27th ICRC, Hamburg 2001, 1125.

[31] A. Karle for the IceCube Coll., Proc. Int. Conf. Neutrino-2002, Munich 2002, 388, and C. Spiering for the IceCube Coll., Proc. VLVNT Workshop, Amsterdam 2003, 21, and astro-ph/0404090

[32] T. Stanev and R. Ulrich for the IceCube Collaboration, astro-ph/0501046

[33] J.Ahrens et al., Astropart. Phys 20 (2004) 507, astro-ph/0305196

[34] C.Spiering, Journ. Phys. G, Nucl. Part. Phys. 29 (2003), astro-ph/030368.

[35] C. DiStefano et al., Phys. Rev. D66 (2002) 063004. 